\title{
Spectra and eigenspaces from regular partitions of Cayley (di)graphs of permutation groups *
}

\author{
C. Dalfó \\ Departament de Matemàtica, Universitat de Lleida \\ Igualada (Barcelona), Catalonia \\ cristina.dalfo@matematica.udl.cat \\ M. A. Fiol \\ Departament de Matemàtiques, Universitat Politècnica de Catalunya \\ Barcelona Graduate School \\ Barcelona, Catalonia \\ miguel.angel.fiol@upc.edu
}

口

\begin{abstract}
In this paper, we present a method to obtain regular (or equitable) partitions of Cayley (di)graphs (that is, graphs, digraphs, or mixed graphs) of permutation groups on $n$ letters. We prove that every partition of the number $n$ gives rise to a regular partition of the Cayley graph. By using representation theory, we also obtain the complete spectra and the eigenspaces of the corresponding quotient (di)graphs. More precisely, we provide a method to find all the eigenvalues and eigenvectors of such (di)graphs, based on their irreducible representations. As examples, we apply this method to the pancake graphs $P(n)$ and to a recent known family of mixed graphs $\Gamma(d, n, r)$ (having edges with and without direction). As a byproduct, the existence of perfect codes in $P(n)$ allows us to give a lower bound for the multiplicity of its eigenvalue -1 .
\end{abstract}

Mathematics Subject Classifications: 05C50.

${ }^{*}$ This research has been partially supported by AGAUR from the Catalan Government under project 2017SGR1087 and by MICINN from the Spanish Government under project PGC2018-095471-B-I00. The research of the first author has also been supported by MICINN from the Spanish Government under project MTM2017-83271-R.

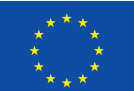

The research of the first author has also received funding from the European Union's Horizon 2020 research and innovation programme under the Marie Skłodowska-Curie grant agreement No 734922 . 
Keywords: Lifted (di)graph, regular partition, spectrum, symmetric group, representation theory, pancake graph, new mixed graph.

\section{Preliminaries}

In this paper, we study the eigenvalues and eigenvectors of Cayley (di)graphs Cay $(G, S)$ (in these, we include graphs, digraphs, and mixed graphs), where $G$ is a subgroup of the symmetric group $S_{n}=\operatorname{Sym}(n)$, and $S$ is the generating set given by some permutations $\pi_{1}, \pi_{2}, \ldots, \pi_{k}$.

Throughout this paper, $\Gamma=(V, E)$ denotes a digraph, which as said before can be a graph, digraph or mixed graph, with vertex set $V$ and arc set $E$. An arc from vertex $u$ to vertex $v$ is denoted by either $u v$ or $u \rightarrow v$. The set of vertices adjacent from a vertex $u \in V$ is denoted by $\Gamma^{+}(u)=\{v \in V: u \rightarrow v\}$. We allow loops (that is, arcs from a vertex to itself), and multiple arcs. A digon is a pair of opposite arcs, $u v$ and $v u$, forming an edge, and is denoted by $u \sim v$. So from now on, and without loss of generality, we refer to $\Gamma$ as a digraph, unless stated otherwise. In particular if $\Gamma$ contains both edges and arcs, it is usually referred to as a mixed (or partially directed) graph. For more details, see the comprehensive survey of Miller and Šráň [17].

If $\Gamma$ has adjacency matrix $A$, its spectrum

$$
\operatorname{sp} \Gamma=\operatorname{sp} A=\left\{\left[\lambda_{0}\right]^{m_{0}},\left[\lambda_{1}\right]^{m_{1}}, \ldots,\left[\lambda_{d}\right]^{m_{d}}\right\},
$$

is constituted by the (possibly complex) distinct eigenvalues with the corresponding algebraic multiplicities $m_{i}$, for $i \in[n]=\{1, \ldots, n\}$.

\subsection{Regular partitions and their spectra}

Let $\Gamma=(V, E)$ be a digraph with adjacency matrix $A$. A partition $\pi=\left(V_{1}, \ldots, V_{m}\right)$ of its vertex set $V$ is called regular (or equitable) whenever, for any $i, j=1, \ldots, m$, the intersection numbers $b_{i j}(u)=\left|\Gamma^{+}(u) \cap V_{j}\right|$, where $u \in V_{i}$, do not depend on the vertex $u$ but only on the subsets (usually called classes or cells) $V_{i}$ and $V_{j}$. In this case, such numbers are simply written as $b_{i j}$, and the $m \times m$ matrix $B=\left(b_{i j}\right)$ is referred to as the quotient matrix of $A$ with respect to $\pi$. This is also represented by the quotient (weighted) digraph $\pi(\Gamma)$ (associated with the partition $\pi$ ), with vertices representing the cells, and there is an arc with weight $b_{i j}$ from vertex $V_{i}$ to vertex $V_{j}$ if and only if $b_{i j} \neq 0$.

The characteristic matrix of a partition $\pi$ is the $n \times m$ matrix $\boldsymbol{S}=\left(s_{u i}\right)$ whose $i$-th column is the characteristic vector of $V_{i}$, that is, $s_{u i}=1$ if $u \in V_{i}$, and $s_{u i}=0$ otherwise. In terms of this matrix, we have the following characterization of regular partitions and their spectra (see Godsil [12]). 
Lemma 1.1 ([12]). Let $\Gamma=(V, E)$ be a digraph with adjacency matrix $A$, and vertex partition $\pi$ with characteristic matrix $S$.

(i) The partition $\pi$ is regular if and only if there exists an $m \times m$ matrix $C$ such that $S C=A S$. Moreover, $C=B$, the quotient matrix of $A$ with respect to $\pi$.

(ii) If $\pi$ is regular and $x$ is an eigenvector of $B$, then $S x$ is an eigenvector of $A$. Consequently, the spectrum of $\pi(\Gamma)$ is contained in the spectrum of $\Gamma$, that is, $\operatorname{sp} B \subseteq \operatorname{sp} A$.

\subsection{Lift digraphs and their spectra}

Given a group $G$ with generating set $S$, a voltage assignment of the base digraph $\Gamma$ is a mapping $\alpha: E \rightarrow S$. The pair $(\Gamma, \alpha)$ is often called a voltage digraph. The lifted digraph (or, simply, lift) $\Gamma^{\alpha}$ is the digraph with vertex set $V\left(\Gamma^{\alpha}\right)=V \times G$ and arc set $E\left(\Gamma^{\alpha}\right)=E \times G$, where there is an arc from the vertex $(u, g)$ to the vertex $(v, h)$ if and only if $u v \in E$ and $h=\alpha(u v) g$. In this case, we refer to a regular lift because of the mapping $\phi: \Gamma^{\alpha} \rightarrow \Gamma$ defined by erasing the second coordinate (that is, $\phi(u, g)=u$ and $\phi(a, g)=a$ for every $u \in N$ and $a \in E)$ is a regular ( $|G|$-fold) covering, in its usual meaning in algebraic topology (see, for instance, Gross and Tucker [13]).

As a particular case of a lifted graph, notice that the Cayley digraph $\operatorname{Cay}(G, S)$ can be seen as a lift of the base digraph $\Gamma$ consisting of a vertex with $|S|$ (directed) loops, each of them having assigned, through $\alpha$, an element of $S$.

To the pair $(\Gamma, \alpha)$, we assign the $k \times k$ base matrix $B$, a square matrix whose rows and columns are indexed by the elements of the vertex set of $\Gamma$, and whose $u v$-th element $B_{u, v}$ is determined as follows: If $a_{1}, \ldots, a_{j}$ is the set of all the arcs of $\Gamma$ emanating from $u$ and terminating at $v$ (not excluding the case $u=v$ ), then

$$
B_{u, v}=\alpha\left(a_{1}\right)+\cdots+\alpha\left(a_{j}\right),
$$

the sum being an element of the complex group algebra $\mathbb{C}(G)$; otherwise, we let $B_{u, v}=0$. Given a unitary irreducible representation of $G, \rho \in \operatorname{Irep}(G)$, of dimension $d_{\rho}$, let $\rho(B)$ be the $d_{\rho} k \times d_{\rho} k$ matrix obtained from $B$ by replacing every entry $B_{u, v} \in \mathbb{C}(G)$ as in (1) by the $d_{\rho} \times d_{\rho}$ matrix

$$
\rho\left(B_{u, v}\right)=\left\{\begin{array}{cc}
\rho\left(\alpha\left(a_{1}\right)\right)+\cdots+\rho\left(\alpha\left(a_{j}\right)\right) & \text { if } B_{u, v} \neq 0 \\
O & \text { otherwise }
\end{array}\right.
$$

where $O$ is the all-zero $d_{\rho} \times d_{\rho}$ matrix.

The following results from Širán̆ and the authors [7] (see also [6]) allow us to compute the spectrum of a (regular) lifted digraph from its associated matrix and the irreducible representations of its corresponding group. For more information on representation theory, see James and Liebeck [15] or Burrow [2]. 
Theorem 1.1 ([7]). Let $\Gamma=(V, E)$ be a base digraph on $k$ vertices, with a voltage assignment $\alpha$ in a group $G$, with $|G|=n$. For every irreducible representation $\rho \in \operatorname{Irep} G$, let $\rho(B)$ be the complex matrix whose entries are given by (2). Then,

$$
\operatorname{sp} \Gamma^{\alpha}=\bigcup_{\rho \in \operatorname{Irep}(G)} d_{\rho} \cdot \operatorname{sp}(\rho(B)) .
$$

The result of Theorem 1.1 can be generalized to deal with the so-called relative voltage assignments and (not necessarily regular) lifts, which are defined as follows. Let $\Gamma=(V, E)$ be the digraph considered above, $G$ a group, and $H$ a subgroup of $G$ of index $n$. Let $G / H$ denote the set of left cosets of $H$ in $G$. Furthermore, let $\beta: E \rightarrow G$ be a mapping defined on every arc $a \in E$. In this context, one calls $\beta$ a voltage assignment in $G$ relative to $H$, or simply a relative voltage assignment. Then, the relative lift $\Gamma^{\beta}$ has vertex set $V^{\beta}=V \times G / H$ and arc set $E^{\beta}=E \times G / H$. Incidence in the lift is given as expected: If $a$ is an arc from a vertex $u$ to a vertex $v$ in $\Gamma$, then for every left coset $J \in G / H$ there is an $\operatorname{arc}(a, J)$ from the vertex $(u, J)$ to the vertex $(v, \beta(a) J)$ in $\Gamma^{\beta}$. Notice that a relative voltage assignment $\beta$ in a group $G$ with subgroup $H$ is equivalent to a regular voltage assignment if and only if $H$ is a normal subgroup of $G$. In such a case, the relative lift $\Gamma^{\beta}$ admits a description in terms of ordinary voltage assignment in the factor group $G / H$, with voltage $\beta(a) H$ assigned to an arc $a \in E$ with original relative voltage $\beta(a)$. In this context, Pavlíková, Širán̆, and the authors [8] proved the following result, which generalizes Theorem 1.1 for relative voltage assignments.

Theorem $1.2([8])$. Let $\Gamma$ be a base digraph of order $k$ and let $\beta$ be a voltage assignment on $\Gamma$ in a group $G$ relative to a subgroup $H$ of index $n$ in $G$. Given an irreducible representation $\rho \in \operatorname{Irep}(G)$, let us consider the matrix $\rho(H)=\sum_{h \in H} \rho(h)$. Then,

$$
\operatorname{sp} \Gamma^{\beta}=\bigcup_{\rho \in \operatorname{Irep}(G)} \operatorname{rank}(\rho(H)) \cdot \operatorname{sp}(\rho(B)),
$$

where the union must be understood for all $\rho \in \operatorname{Irep}(G)$ such that $\operatorname{rank}(\rho(H)) \neq 0$.

\subsection{The pancake graphs}

To illustrate our results, we use two families of Cayley graphs: The pancake graphs and a new family of mixed graphs introduced in [5], which can be seen as a generalization of both the pancake graphs and the cycle prefix digraphs. Let us first introduce the pancake graphs, together with some of their basic properties.

The $n$-dimensional pancake graph, proposed by Dweighter [9] (see also Akers and Krishnamuthy [1]), and denoted by $P(n)$, is a graph with the vertex set $V(P(n))=$ $\left\{x_{1} x_{2} \ldots x_{n} \mid x_{i} \in[n], x_{i} \neq x_{j}\right.$ for $\left.i \neq j\right\}$. Its adjacencies are as follows: 


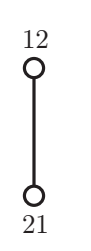

(a)

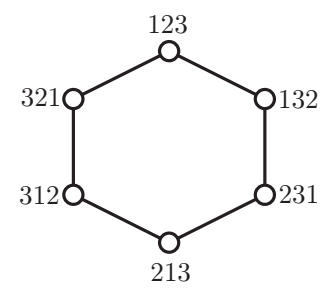

(b)

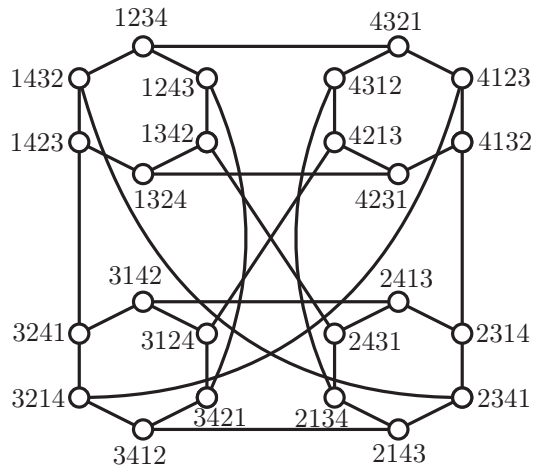

$(c)$

Figure 1: Pancakes graphs: (a) $P(2),(b) P(3)$, and (c) $P(4)$.

\begin{tabular}{|c|ccccccccccccccccc|}
\hline$n$ & 1 & 2 & 3 & 4 & 5 & 6 & 7 & 8 & 9 & 10 & 11 & 12 & 13 & 14 & 15 & 16 & 17 \\
\hline$k$ & 0 & 1 & 3 & 4 & 5 & 7 & 8 & 9 & 10 & 11 & 13 & 14 & 15 & 16 & 17 & 18 & 19 \\
\hline
\end{tabular}

Table 1: The known values of the diameter $k$ of the pancake graph $P(n)$.

$$
x_{1} x_{2} \ldots x_{n} \sim\left\{\begin{array}{c}
x_{1} \ldots x_{n-2} x_{n} x_{n-1} \\
x_{1} \ldots x_{n-3} x_{n} x_{n-1} x_{n-2}, \\
x_{1} \ldots x_{n-4} x_{n} x_{n-1} x_{n-2} x_{n-3} \\
\vdots \\
x_{n} x_{n-1} \ldots x_{2} x_{1} .
\end{array}\right.
$$

The pancake graph $P(n)$ is a vertex-transitive $(n-1)$-regular graph with $n$ ! vertices. It is a Cayley graph Cay $(G, S)$, where $G$ is the symmetric group $\operatorname{Sym}(n)$ and the generating set $S$ corresponds to the permutations of $x_{1}, x_{2}, \ldots, x_{n}$ given by (3). As examples, the pancakes graphs $P(2), P(3)$, and $P(4)$ are shown in Figure 1 .

The exact diameters $k=k(n)$ of $P(n)$ are only known for $n \leq 17$, as shown in Table 1 (see Cibulka [3] and Sloane [18]). The best results to our knowledge were given by Gates and Papadimitriou [11], who proved that

$$
\frac{17}{16} n \leq k(n) \leq \frac{5 n+5}{3}
$$

and by Heydari and Sudborough [14], who improved the lower bound to

$$
k(n) \geq \frac{15}{14} n .
$$




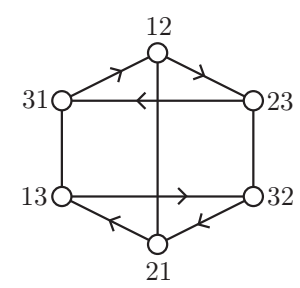

$(a)$

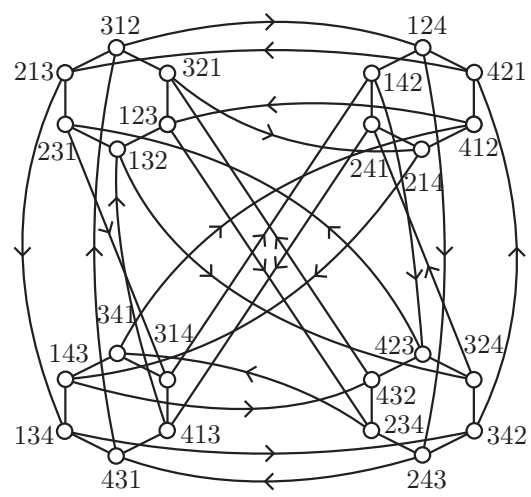

(b)

Figure 2: New mixed graphs: $(a) \Gamma(2,2,1)$ and $(b) \Gamma(3,3,2)$.

\subsection{The new mixed graphs $\Gamma(d, n, r)$}

Recently, the pancake graphs, together with the cyclic prefix digraphs, were used by the first author [5] to propose a new general family of mixed graphs.

The definition of these new mixed graphs is as follows.

Definition 1. Given the integers $n \geq 2$ and $d, r \geq 1$, with $r<n \leq d+1$, the mixed graph $\Gamma(d, n, r)$ has as vertex set the $n$-permutations of the $d+1$ symbols $1,2, \ldots, d, d+1$. Moreover, a vertex $x_{1} x_{2} \ldots x_{n}$ is adjacent, through edges, to the $r$ vertices

$$
x_{1} x_{2} \ldots x_{n} \sim\left\{\begin{array}{c}
x_{1} x_{2} \ldots x_{n-2} x_{n} x_{n-1} \\
x_{1} x_{2} \ldots x_{n-3} x_{n} x_{n-1} x_{n-2} \\
\vdots \\
x_{1} \ldots x_{n-r-1} x_{n} x_{n-1} \ldots x_{n-r}
\end{array}\right.
$$

and adjacent, through arcs, to the $z=d-r$ vertices

$$
x_{1} x_{2} \ldots x_{n} \rightarrow\left\{\begin{array}{c}
x_{2} x_{3} \ldots x_{n} y, \quad y \neq x_{i}, i=1, \ldots, n \\
x_{1} \ldots x_{n-r-2} x_{n-r} \ldots x_{n} x_{n-r-1} \\
x_{1} \ldots x_{n-r-3} x_{n-r-1} \ldots x_{n} x_{n-r-2} \\
\vdots \\
x_{2} \ldots x_{n} x_{1}
\end{array}\right\}(n-r-1 \text { vertices }) .
$$

Thus, the number of vertices of the mixed graph $\Gamma(d, n, r)$ is the number of $n$-permutations of $d+1$ elements, $N=\frac{(d+1) !}{(d+1-n) !}$. Moreover, $\Gamma(d, n, r)$ is a totally $(r, z)$-regular mixed graph, and it is also vertex-transitive. In particular, if $n=d+1$ and $r=d$, then $\Gamma(n-1, n, n-1)$ is the pancake graph $P(n)$; and if $r=1$, then $\Gamma(d, n, 1)$ coincides with the so-called cycle prefix digraph $\Gamma_{d}(n)$ (notice that in this case, we require that $d \geq n$ ), 

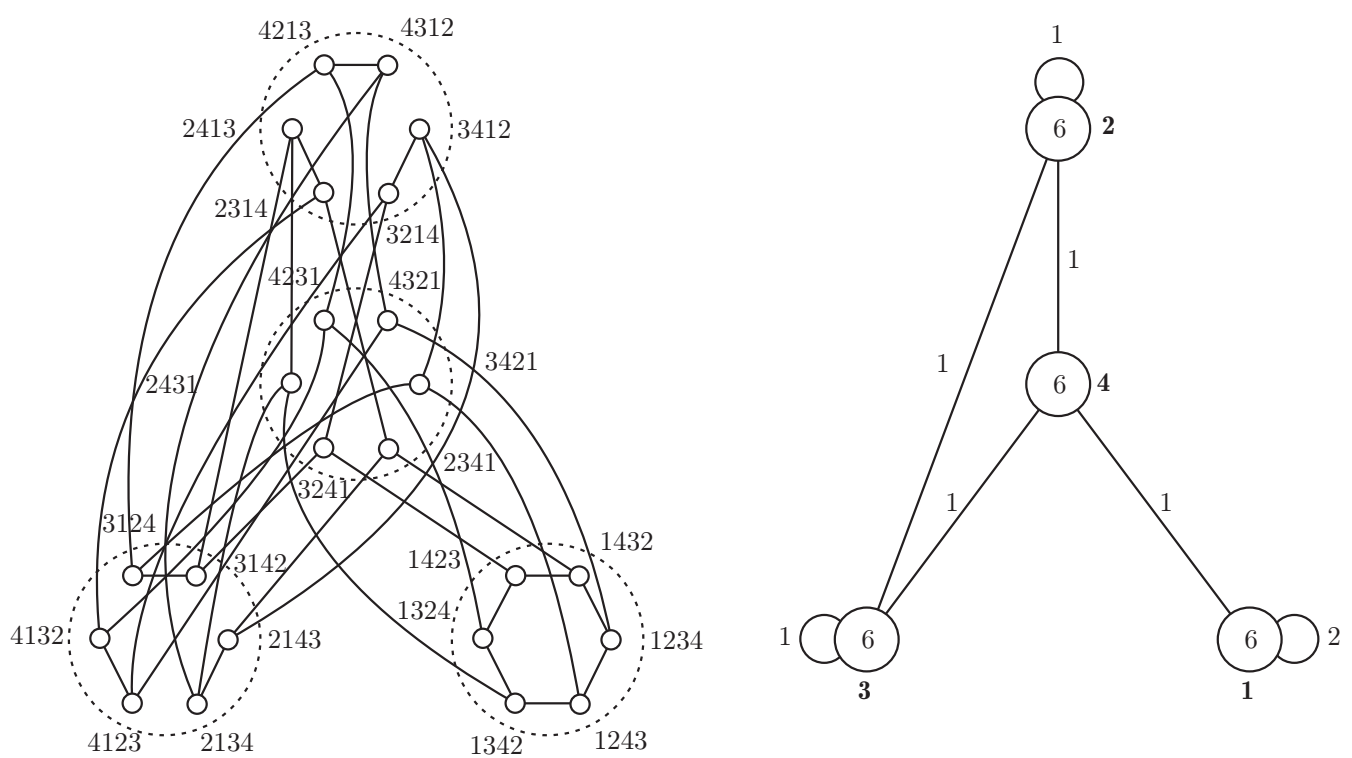

Figure 3: A regular partition of the pancake graph $P(4)$ and its quotient graph. In boldface there is the numbering of the vertices (or classes).

see Faber, Moore, and Chen [10] or Comellas and Fiol [4. As examples, the new mixed graphs $\Gamma(2,2,1)$ and $\Gamma(3,3,2)$ are depicted in Figure 2 .

\section{Regular partitions of vertices from number partitions}

Given a permutation $\pi:[n] \rightarrow[n]$, we denote by $P(\pi)=\left(p_{i j}\right)$ the $n \times n$ permutation matrix with entries $p_{i j}=1$ if $\pi(i)=j$, and 0 otherwise (that is, the so-called column representation).

Proposition 2.1. Let $\Gamma=\operatorname{Cay}(G, S)$ be a Cayley digraph, where $G$ is a subgroup of the symmetric group $\operatorname{Sym}(n)$ and its generating set $S$ is given by the permutations $\left\{\pi_{1}, \pi_{2}, \ldots\right.$, $\left.\pi_{k}\right\}$. Then, $\Gamma$ has a regular partition $\beta$ with quotient matrix $B=\sum_{i=1}^{k} P\left(\pi_{i}\right)$.

Proof. Let us show that the cells of the regular partition $\beta$ are the sets $V_{i}$, for $i=1, \ldots, n$, constituted by the permutations with a given digit, say 1 , in the fixed position $i$, that is $V_{i}=\{\pi \in S: \pi(i)=1\}$. Indeed, if $u \in V_{i}$, the number of vertices $\left|\Gamma^{+}(u) \cap V_{j}\right|$ (adjacent from $u$ and belonging to $V_{j}$ ) corresponds to the number of the permutations in $S$ that sends 1 from the position $i$ to position $j$. This is precisely the $(i, j)$-entry of the matrix $B$, which is independent of $u$.

As a corollary, we have a handy way of obtaining some of the eigenvalues of $\Gamma$ since, by Lemma $1.1(i i), \operatorname{sp} B \subset \operatorname{sp} A(\Gamma)$. 
Example (Pancake graph $P(4))$. Consider the pancake graph $P(4)$ as the Cayley graph Cay $\left(S_{4}, S\right)$ with $S=\{(34),(24),(14)(23)\}$. Then, the sum of the corresponding permutation matrices $B=P((34))+P((24))+P((14)(23))$ turns out to be

$$
B=\left(\begin{array}{llll}
2 & 0 & 0 & 1 \\
0 & 1 & 1 & 1 \\
0 & 1 & 1 & 1 \\
1 & 1 & 1 & 0
\end{array}\right) .
$$

According to Proposition 2.1, this is the quotient matrix of a regular partition of $P(4)$, as shown in Figure 3, together with its quotient graph. Notice that, as claimed, each class of vertices contains all the permutations with 1 in a fixed position. Moreover, $\operatorname{sp} B=$ $\{3,2,0,-1\}$, a part of the spectrum of $P(4)$ that, as we show in Section 3 , it is

$$
\operatorname{sp} P(4)=\left\{[3]^{1},[2]^{5},\left[\frac{-1+\sqrt{17}}{2}\right]^{3},[0]^{5},\left[\frac{-1-\sqrt{17}}{2}\right]^{3},[-1]^{4},[-2]^{3}\right\} .
$$

Example (New mixed graph $\Gamma(3,3,2))$. Consider the new mixed graph $\Gamma(3,3,2)$ as the Cayley graph Cay $\left(S_{4}, S\right)$ with $S=\{(34),(24),(2341)\}$. Now, the sum of the corresponding permutation matrices $B=P((34))+P((24))+P((2341))$ is

$$
B=\left(\begin{array}{llll}
2 & 0 & 0 & 1 \\
1 & 1 & 0 & 1 \\
0 & 1 & 1 & 1 \\
0 & 1 & 2 & 0
\end{array}\right),
$$

that corresponds to the quotient matrix of the regular partition shown in Figure 4 . Moreover, $\operatorname{sp} B=\left\{[3]^{1},[1]^{2},[-1]^{2}\right\}$. As expected, $\operatorname{sp} B \subset \operatorname{sp} \Gamma(3,3,2)$, since, as shown in Section 3.

$$
\operatorname{sp} \Gamma(3,3,2)=\left\{[3]^{1},[\sqrt{3}]^{2},[1]^{9},[-1]^{9},[-\sqrt{3}]^{2},[-3]^{1}\right\} .
$$

Note that this spectrum is symmetric, in concordance with the fact that $\Gamma(3,3,2)$ is a bipartite (mixed) graph.

The result of the previous examples can be generalized to obtain some eigenvalues and their associated eigenvectors of the whole family of the pancake graphs $P(n)$ and the new mixed graphs $\Gamma(n, n, n-1)$, as shown in the following results.

Proposition 2.2. The matrix $B_{n}=\sum_{i=1}^{n} P\left(\pi_{i}\right)$ of the pancake graph $P(n)$ is the sum $B_{n}=D_{n}+T_{n}$, where $D_{n}=\operatorname{diag}(n-2, n-1, \ldots, 0,-1)$ and $T_{n}$ is the 'lower anti-triangular matrix' with entries $\left(T_{n}\right)_{i j}=1$ if $i+j \geq n+1$, and $\left(T_{n}\right)_{i j}=0$ otherwise, with spectrum

$$
\operatorname{sp} B_{n}=\{n-1, n-2, \ldots, 0,-1\} \backslash\{\lfloor(n / 2)-1\rfloor\}
$$

(all the eigenvalues with multiplicity one). Moreover, their associated eigenvectors are, respectively, the all-1 vector $(1,1, \ldots, 1)^{\top}$,

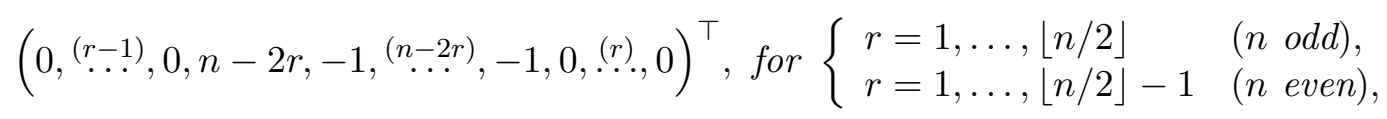



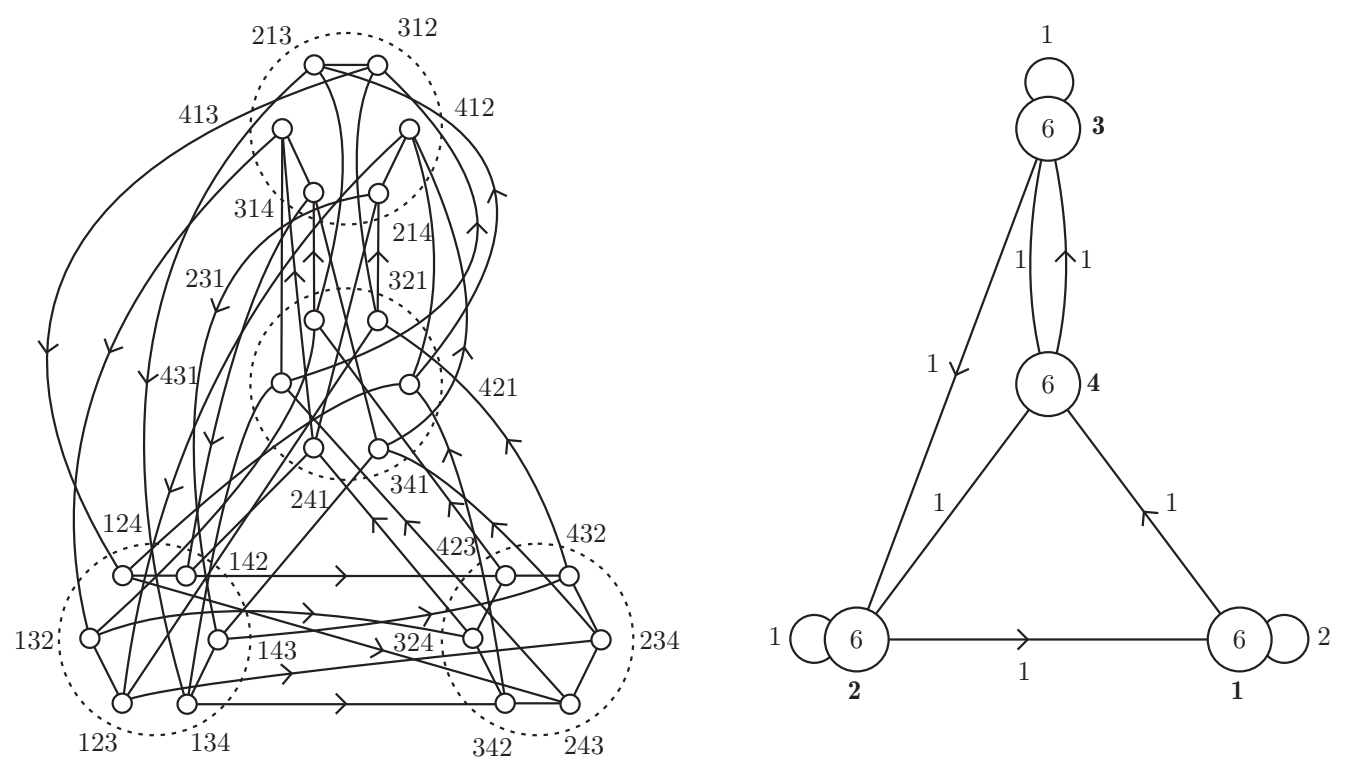

Figure 4: The new mixed graph $\Gamma(3,3,2)$ drawn as a regular partition and its quotient graph. In boldface there is the numbering of the vertices.

and

$$
(0, \stackrel{(r-1)}{\cdots}, 0,-1, \stackrel{(n-2 r+1)}{\cdots},-1, n-2 r+1,0, \stackrel{(r-1)}{\cdots}, 0)^{\top}, \text { for } r=\lfloor n / 2\rfloor, \ldots, \ldots, 1 .
$$

Proof. First, it is straightforward to check that the matrix $B_{n}$ is as claimed. We just have to compute the sum of the involved permutation matrices. For example, for $n=5$, we get

$$
\begin{aligned}
& B_{5}=\left(\begin{array}{lllll}
1 & 0 & 0 & 0 & 0 \\
0 & 1 & 0 & 0 & 0 \\
0 & 0 & 1 & 0 & 0 \\
0 & 0 & 0 & 0 & 1 \\
0 & 0 & 0 & 1 & 0
\end{array}\right)+\left(\begin{array}{lllll}
1 & 0 & 0 & 0 & 0 \\
0 & 1 & 0 & 0 & 0 \\
0 & 0 & 0 & 0 & 1 \\
0 & 0 & 0 & 1 & 0 \\
0 & 0 & 1 & 0 & 0
\end{array}\right)+\left(\begin{array}{ccccc}
1 & 0 & 0 & 0 & 0 \\
0 & 0 & 0 & 0 & 1 \\
0 & 0 & 0 & 1 & 0 \\
0 & 0 & 1 & 0 & 0 \\
0 & 1 & 0 & 0 & 0
\end{array}\right)+\left(\begin{array}{lllll}
0 & 0 & 0 & 0 & 1 \\
0 & 0 & 0 & 1 & 0 \\
0 & 0 & 1 & 0 & 0 \\
0 & 1 & 0 & 0 & 0 \\
1 & 0 & 0 & 0 & 0
\end{array}\right) \\
& =\left(\begin{array}{lllll}
3 & 0 & 0 & 0 & 1 \\
0 & 2 & 0 & 1 & 1 \\
0 & 0 & 2 & 1 & 1 \\
0 & 1 & 1 & 1 & 1 \\
1 & 1 & 1 & 1 & 0
\end{array}\right)=\left(\begin{array}{lllll}
3 & 0 & 0 & 0 & 0 \\
0 & 2 & 0 & 0 & 0 \\
0 & 0 & 1 & 0 & 0 \\
0 & 0 & 0 & 0 & 0 \\
0 & 0 & 0 & 0 & -1
\end{array}\right)+\left(\begin{array}{lllll}
0 & 0 & 0 & 0 & 1 \\
0 & 0 & 0 & 1 & 1 \\
0 & 0 & 1 & 1 & 1 \\
0 & 1 & 1 & 1 & 1 \\
1 & 1 & 1 & 1 & 1
\end{array}\right)=D_{5}+T_{5} .
\end{aligned}
$$

Concerning the eigenpairs, in the case that $n$ is even, let us check that, for every $r=1, \ldots, n / 2$,

$$
v_{r}=(0, \stackrel{(r-1)}{\cdots}, 0, n-2 r,-1, \stackrel{(n-2 r)}{\cdots},-1,0, . \stackrel{(r)}{\cdot}, 0)^{\top}
$$

is an eigenvector with eigenvalue $\lambda_{r}=n-r-1$ : 


$$
\begin{aligned}
& B_{n} v_{k}=D_{n} v_{k}+T_{n} v_{k} \\
& =(0, \stackrel{(r-1)}{\cdots}, 0,(n-2 r)(n-r-1),-(n-r-2),-(n-r-3), \ldots,-(r-1), 0, . \stackrel{(r)}{\cdot}, 0)^{\top} \\
& +(0, \stackrel{(r)}{.}, 0,-1,-2, \ldots,-(n-2 r), 0, \stackrel{(r)}{.}, 0)^{\top} \\
& =\left(0, \stackrel{(r-1)}{\cdots}, 0,(n-2 r) \lambda_{r},-\lambda_{r}+1,-\lambda_{r}+2,-\lambda_{r}+3, \ldots,-\lambda_{r}+(n-2 r), 0, . \stackrel{(r)}{\cdot}, 0\right)^{\top} \\
& +(0, \stackrel{(r)}{.}, 0,-1,-2, \ldots,-(n-2 r), 0, \stackrel{(r)}{.}, 0)^{\top} \\
& =\lambda_{r}(0, \stackrel{(r-1)}{\cdots}, 0, n-2 r,-1, \stackrel{(n-2 r)}{\cdots},-1,0, \stackrel{(r)}{\cdot}, 0)^{\top}=\lambda_{r} v_{r}
\end{aligned}
$$

The other eigenpairs and the case for odd $n$ can be proved analogously.

For example, for the case $n=5$, we obtain sp $B_{5}=\{4,3,2,0,-1\}$, with corresponding matrix of (column) eigenvectors

$$
\left(\begin{array}{rrrrr}
1 & 3 & 0 & 0 & -1 \\
1 & -1 & 1 & -1 & -1 \\
1 & -1 & -1 & -1 & -1 \\
1 & -1 & 0 & 2 & -1 \\
1 & 0 & 0 & 0 & 4
\end{array}\right) .
$$

Looking at last column, Lemma 1.1(ii) implies that $P(n)$ has the eigenvalue -1 whose eigenvector has entries -1 for the vertices of the classes $1,2, \ldots, n-1$, and entries $n-1$ for the vertices of the class $n$ (permutations with last symbol 1, as shown in Figure 3 for the case $n=4)$. In this situation, it is known that the graph has a perfect code or efficient dominating set $C$ (that is, $C$ is an independent vertex set, and each vertex not in $C$ is adjacent to exactly one vertex in $C$ ). In other words, to each perfect code $C$ corresponds a $(-1)$-eigenvector as described. See, for instance, Godsil [12]. Then, by using the result by Konstantinova [16, Thm. 1], who proved that the pancake graph $P(n)$ contains exactly $n$ perfect codes (the sets of vertices with the same last symbol), we get the following result. First, recall that a circulant matrix $\operatorname{circ}\left(a_{1}, a_{2}, \ldots, a_{n}\right)$ has first row $a_{1}, a_{2}, \ldots, a_{n}$ and, for $i=2, \ldots, n$, its $i$-th row is obtained from the $(i-1)$-th row by cyclically shifting it to the right one position.

Lemma 2.1. The pancake graph $P(n)$ has eigenvalue -1 with multiplicity $m(-1) \geq n-1$.

Proof. Each of the $n$ different perfect codes induces a regular partition with quotient matrix having a $(-1)$-eigenvector as above. Then, $\operatorname{since} \operatorname{rank} \operatorname{circ}(n-1,-1, \stackrel{(n-1)}{\cdots},-1)=$ $n-1$, we conclude that $n-1$ of such eigenvectors are independent.

In the case of the new mixed graph $\Gamma(n, n, n-1)$, we have the following result. 
Proposition 2.3. The matrix $B_{n}^{\prime}=\sum_{i=1}^{n} P\left(\pi_{i}\right)$ of the new mixed graph $\Gamma(n, n, n-1)$, $n \geq 3$, is the sum $B_{n}^{\prime}=D_{n}+C_{n}+T_{n}^{\prime}$, where $D_{n}=\operatorname{diag}(n-2, n-1, \ldots, 0,-1), C_{n}$ is the circulant matrix $\operatorname{circ}(0,1,0, \ldots, 0)$, and $T_{n}^{\prime}$ is the 'lower anti-triangular matrix' with entries $\left(T_{n}^{\prime}\right)_{i j}=1$ if $i+j>n+1$ and $\left(T_{n}^{\prime}\right)_{i j}=0$ otherwise, with eigenvalues $\{n-1, n-3,-1\} \subset$ $\operatorname{sp} B_{n}^{\prime}$.

Proof. Again, by computing the sum of the involved permutation matrices, it is easy to check that the matrix $B_{n}^{\prime}$ is as claimed. For example, for $n=5$, we get

$$
\begin{aligned}
& B_{5}^{\prime}=\left(\begin{array}{ccccc}
1 & 0 & 0 & 0 & 0 \\
0 & 1 & 0 & 0 & 0 \\
0 & 0 & 1 & 0 & 0 \\
0 & 0 & 0 & 0 & 1 \\
0 & 0 & 0 & 1 & 0
\end{array}\right)+\left(\begin{array}{ccccc}
1 & 0 & 0 & 0 & 0 \\
0 & 1 & 0 & 0 & 0 \\
0 & 0 & 0 & 0 & 1 \\
0 & 0 & 0 & 1 & 0 \\
0 & 0 & 1 & 0 & 0
\end{array}\right)+\left(\begin{array}{ccccc}
1 & 0 & 0 & 0 & 0 \\
0 & 0 & 0 & 0 & 1 \\
0 & 0 & 0 & 1 & 0 \\
0 & 0 & 1 & 0 & 0 \\
0 & 1 & 0 & 0 & 0
\end{array}\right)+\left(\begin{array}{lllll}
0 & 0 & 0 & 0 & 1 \\
0 & 1 & 0 & 0 & 0 \\
0 & 0 & 1 & 0 & 0 \\
0 & 0 & 0 & 1 & 0 \\
0 & 0 & 0 & 0 & 1
\end{array}\right) \\
& =\left(\begin{array}{lllll}
3 & 0 & 0 & 0 & 1 \\
1 & 2 & 0 & 0 & 1 \\
0 & 1 & 1 & 1 & 1 \\
0 & 0 & 2 & 1 & 1 \\
0 & 1 & 1 & 2 & 0
\end{array}\right)=\left(\begin{array}{ccccc}
3 & 0 & 0 & 0 & 0 \\
0 & 2 & 0 & 0 & 0 \\
0 & 0 & 1 & 0 & 0 \\
0 & 0 & 0 & 0 & 0 \\
0 & 0 & 0 & 0 & -1
\end{array}\right)+\left(\begin{array}{lllll}
0 & 0 & 0 & 0 & 1 \\
0 & 1 & 0 & 0 & 0 \\
0 & 0 & 1 & 0 & 0 \\
0 & 0 & 0 & 1 & 0 \\
0 & 0 & 0 & 0 & 1
\end{array}\right)+\left(\begin{array}{lllll}
0 & 0 & 0 & 0 & 0 \\
0 & 0 & 0 & 0 & 1 \\
0 & 0 & 0 & 1 & 1 \\
0 & 0 & 1 & 1 & 1 \\
0 & 1 & 1 & 1 & 1
\end{array}\right) \\
& =D_{5}+C_{5}+T_{5}^{\prime} \text {. }
\end{aligned}
$$

Concerning the eigenvalues, it is readily checked the vectors $(1,1, \ldots, 1)^{\top}$ and $(-1, \ldots,-1$, $n-1)^{\top}$ are eigenvectors of $B_{n}^{\prime}$ with eigenvalues $n-1$ and -1 respectively. Alternatively, to prove that $-1 \in \operatorname{sp} B_{n}^{\prime}$, we can also check that $\operatorname{det}\left(-I-B_{n}^{\prime}\right)=(-1)^{n} \operatorname{det}\left(I+B_{n}^{\prime}\right)=0$. Note that this holds since $n$ times the last column of $I+B_{n}^{\prime}$ is the sum of the other columns. For instance, for $n=5$, we get

$$
B_{5}^{\prime}+I=\left(\begin{array}{ccccc}
4 & 0 & 0 & 0 & 1 \\
1 & 3 & 0 & 0 & 1 \\
0 & 1 & 2 & 1 & 1 \\
0 & 0 & 2 & 2 & 1 \\
0 & 1 & 1 & 2 & 1
\end{array}\right)
$$

Finally, $n-3$ is an eigenvalue of $B_{n}^{\prime}$ since.the first two rows of $(n-3) I-B_{n}^{\prime}$ are equal. Namely, $(-1,0, \ldots, 0,-1)$.

In fact, the first statments of Propositions 2.1 and 2.3 are particular cases of the following result. Let $\mathcal{P} \mathcal{R}=P R_{n}^{n_{1}, \ldots, n_{r}}$ denote the set of permutations with repetitions of $r$ symbols $a, b, \ldots$, where $a$ is repeated $n_{1}$ times, $b$ is repeated $n_{2}$ times, etc. Thus, $|\mathcal{P R}|=\frac{n !}{n_{1} ! \cdots n_{r} !}$.

Theorem 2.1. Let $\Gamma=\operatorname{Cay}(G, S)$ be a Cayley digraph, where $G$ is a subgroup of the symmetric group $\operatorname{Sym}(n)$ and its generating set $S$ is given by the permutations $\left\{\pi_{1}, \pi_{2}, \ldots, \pi_{k}\right\}$. For any partition $n_{1}+n_{2}+\cdots+n_{r}=n$, there is a regular partition of $\Gamma$ with quotient matrix $B$ indexed by the elements of $\mathcal{P} \mathcal{R}$, and for every $\sigma, \tau \in \mathcal{P} \mathcal{R}$ the entry $(B)_{\sigma \tau}$ is the number (possibly zero) of permutations in $S$ that, acting on the symbol positions, map $\sigma$ into $\tau$. 

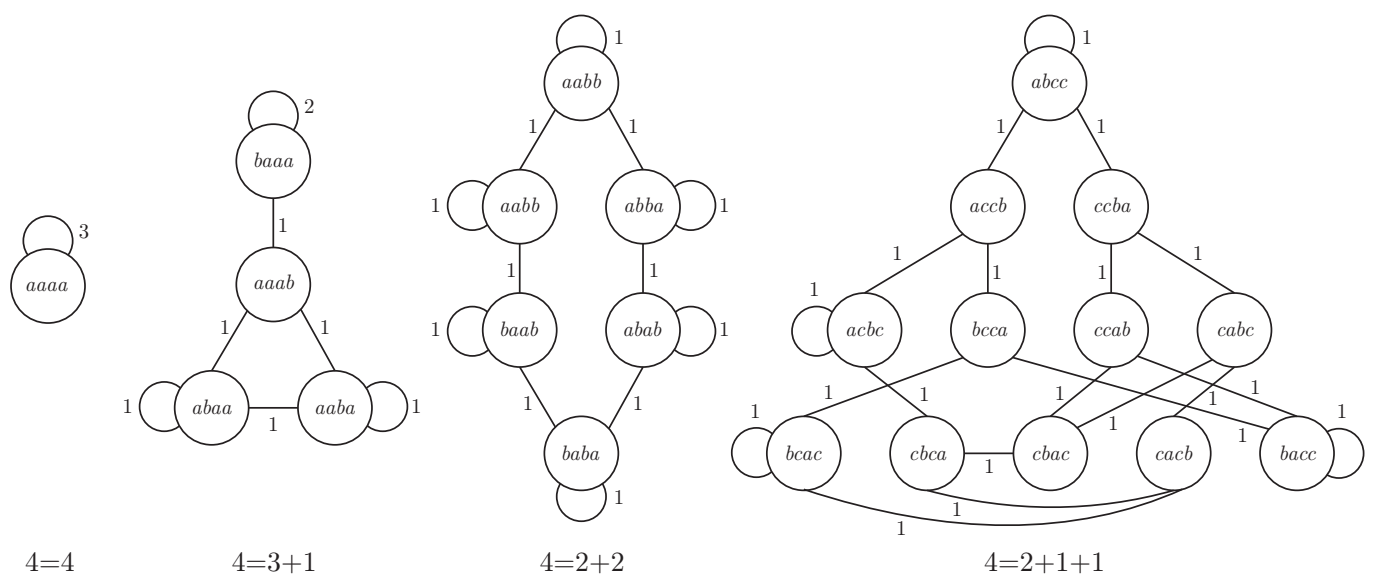

Figure 5: The regular partitions of $P(4)$ corresponding to the number partitions of 4 .

Proof. Given the partition $n_{1}+n_{2}+\cdots+n_{r}=n$, we define the onto mapping $\phi:[n] \rightarrow$ $\left\{a_{1}, \ldots a_{r}\right\}$ such that $\left|\phi^{-1}\left(a_{i}\right)\right|=n_{i}$, for $i=1, \ldots, r$. Given a permutation $\pi \in \operatorname{Sym}(n)$, let $\phi \cdot \pi$ be the permutation with repetition in $\mathcal{P} \mathcal{R}$ with $i$-th symbol $(\phi \cdot \pi)(i)=\phi(\pi(i))$, for $i=1, \ldots, n$. If we let $\pi$ to act on the symbol positions (composition of permutations $g h$ being read from left to right), then we can also define the permutation with repetition $\pi \cdot \phi$ such that $(\pi \cdot \phi)(i)=\pi(\phi(i))=\phi(\pi(i))$ for $i=1, \ldots, n$ and, hence, $\phi \cdot \pi=\pi \cdot \phi$. Also, it is clear that, for any $g, h \in G,(g h) \cdot \phi=g \cdot(h \cdot \phi)$. Let $\phi(G)$ be the set of distinct permutations in $\mathcal{P} \mathcal{R}$ of the form $\phi \cdot g$ for some $g \in G$. Now we claim that $\Gamma$ has a regular partition $\phi^{*}$, where each class $V_{\sigma}$ is represented by an element $\sigma \in \phi(G)$. More precisely, $V_{\sigma}=\{g \in G: \phi \cdot g=\sigma\}$. Indeed, if $\phi \cdot g=\phi \cdot g^{\prime}$ and $g \rightarrow \pi g$ for some $\pi \in S$, we have

$$
\begin{aligned}
\phi \cdot(\pi g) & =(\pi g) \cdot \phi=\pi \cdot(g \cdot \phi)=\pi \cdot(\phi \cdot g) \\
& =\pi \cdot\left(\phi \cdot g^{\prime}\right)=\pi \cdot\left(g^{\prime} \cdot \phi\right)=\left(\pi g^{\prime}\right) \cdot \phi=\phi \cdot\left(\pi g^{\prime}\right) .
\end{aligned}
$$

Thus, $\phi$ can be interpreted as a homomorphism from $\Gamma$ to its quotient digraph $\phi^{*}(\Gamma)$ that preserves the 'colors' (generators) of the arcs. The corresponding quotient matrix $B$ is then indexed by elements of $\phi(G) \subset \mathcal{P} \mathcal{R}$, with entries $(B)_{\sigma \tau}$ for every $\sigma, \tau \in \mathcal{P} \mathcal{R}$, as claimed.

Example (Pancake graph $P(4))$. Consider the pancake graph $P(4)$. In this case, we have the following partitions: $4,3+1,2+2,2+1+1$, and $1+1+1+1$. According to Theorem 2.1, these partitions yield the regular partitions of $P(4)$ in Figure 5, with number of classes $P R_{4}^{4}=1, P R_{4}^{3,1}=4, P R_{4}^{2,2}=6$, and $P R_{4}^{2,1,1}=12$, respectively. Note that the classes are identified with the corresponding permutations with repetition of the symbols $a, b, c$. Besides, observe that the case of the previous example of $P(4)$ corresponds to the partition $3+1$. Note that the graph associated with the partition $1+1+1+1$ is the whole graph $P(4)$, with number of classes (that is, number of vertices) $P R_{4}^{1,1,1,1}=24$ (see Figure 1(c)). 

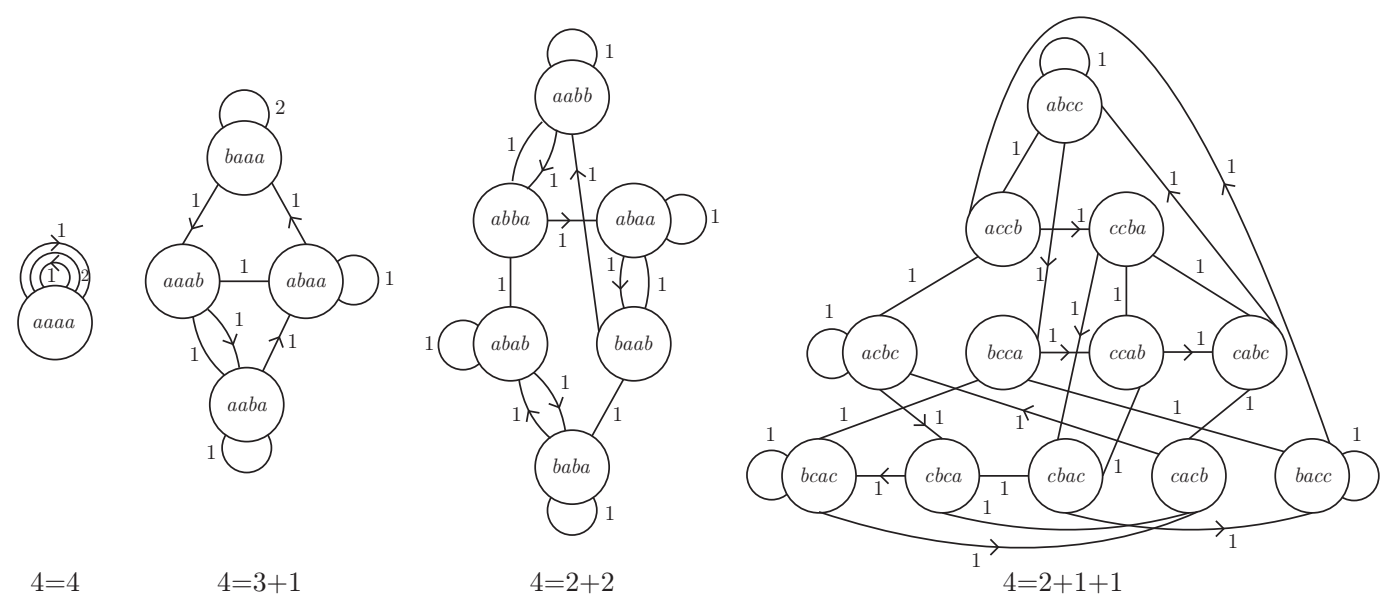

Figure 6: The regular partitions of $\Gamma(3,3,2)$ corresponding to the number partitions of 4 .

Example (New mixed graph $\Gamma(3,3,2)$ ). Consider the new mixed graph $\Gamma(3,3,2)$. We have the partitions $4,3+1,2+2,2+1+1$, and $1+1+1+1$ again. These partitions give the regular partitions of $\Gamma(3,3,2)$ in Figure 6 , with the same number of classes that in the previous example of $P(4)$. The classes are again identified with the corresponding permutations with repetition of the symbols $a, b, c$. Note that the case of the previous example of $\Gamma(3,3,2)$ corresponds to the partition $3+1$. The graph associated with the partition $1+1+1+1$ is the whole graph $\Gamma(3,3,2)$ on 24 vertices (see Figure $2(b)$ ).

\section{The spectra of quotient digraphs}

In the previous section, we found some eigenvalues, eigenvectors, and regular partitions for whole families of digraphs. In this section, we give a method to find the whole spectrum of a Cayley digraph (on a permutation group) and their quotient digraphs associated with the corresponding partitions. We again illustrate the obtained results by using the previous examples: the pancake graph $P(4)$ and the new mixed graph $\Gamma(3,3,2)$.

Our results are based on the following lemma, which allows us to apply Theorem 1.2 .

Lemma 3.1. Let $\Gamma=\operatorname{Cay}(G, S)$ be a Cayley digraph, where $G$ is a subgroup of the symmetric group $\operatorname{Sym}(n)$ with generating set $S=\left\{\pi_{1}, \pi_{2}, \ldots, \pi_{k}\right\}$. For a given partition $n_{1}+n_{2}+\cdots+n_{r}=n$ induced by the mapping $\phi$, the quotient digraph $\phi^{*}(\Gamma)$ is isomorphic to the relative lift $\Gamma^{\beta}$ with base digraph a singleton with arcs $a_{1}, \ldots, a_{k}$, group $G$, relative voltage assignment $\beta$ defined by $\beta\left(a_{i}\right)=\pi_{i}$ for $i=1, \ldots, k$, and stabilizer subgroup

$$
H=\operatorname{Stab}_{G}\left(V_{1}\right) \cap \cdots \cap \operatorname{Stab}_{G}\left(V_{r}\right),
$$

where $V_{1} \cup \cdots \cup V_{r}$ is a partition of $[n]$ with $\left|V_{i}\right|=n_{i}$ for $i=1, \ldots, r$. 
Proof. Let $\phi$ be the mapping defined in the proof of Theorem 2.1. Let $e$ be the identity element of $G$, and assume that $\phi \cdot e$ is the permutation with repetition $\varepsilon=a_{1}\left(n_{1}\right)$. $a_{1} \ldots a_{r} \stackrel{\left(n_{r}\right)}{.} a_{r}$. Then, $H$ is constituted by all the elements $h \in G$ such that $\phi \cdot h=\varepsilon$ and, in general, each left coset of $H$ is of the form

$$
g H=\{h \in G: \phi \cdot h=\sigma\} \quad \text { if } \phi \cdot g=\sigma .
$$

Thus, $g H$ corresponds to the class, or vertex of $\phi^{*}(\Gamma), V_{\sigma}=\{g \in G: \phi \cdot g=\sigma\}$ with $\sigma \in \phi(G)$. Moreover, $V_{\sigma}$ is adjacent to $V_{\tau}$ through an arc with 'color' $\pi \in S$ if $\tau=$ $\phi \cdot(\pi g)=\pi \cdot \sigma$ (where the second equality comes from $(10)$ ). Consequently, in the relative lift $\Gamma^{\beta}$, the vertex $g H$ is adjacent, through the arc with 'color' $\pi$, to $\pi g H$. This proves the claimed isomorphism.

Example (Pancake graph $P(4))$. Consider the case of the pancake graph $P(4)$ again. We begin computing the spectrum of the whole graph by using Theorem 1.1. We obtained from SageMath the matrices of the irreducible representations of $S_{4}$, that is, $\rho_{1}$ (partition $4=4), \rho_{2}$ (partition $\left.4=3+1\right), \rho_{3}$ (partition $\left.4=2+2\right), \rho_{4}$ (partition $\left.4=2+1+1\right)$, and $\rho_{5}$ (partition $\left.4=1+1+1+1\right)$ shown in Table 2. related to the permutations $a=1243$, $b=1432$, and $c=4321$. Then, from Theorem 1.1, the spectrum of $P(4)$ is the union of the following spectra (Note that the dimension of the matrices gives the multiplicities of the corresponding eigenvalues).

(i) $1 \cdot \operatorname{sp} \rho_{1}(B)=\left\{[3]^{1}\right\}$,

(ii) $3 \cdot \operatorname{sp} \rho_{2}(B)=\left\{[2]^{3},[0]^{3},[-1]^{3}\right\}$,

(iii) $2 \cdot \operatorname{sp} \rho_{3}(B)=\left\{[2]^{2},[0]^{2}\right\}$,

(iv) $\left.3 \cdot \operatorname{sp} \rho_{4}(B)=\left\{\left[\frac{-1+\sqrt{17}}{2}\right]^{3},[-2]^{3}, \frac{-1-\sqrt{17}}{2}\right]^{3}\right\}$,

(v) $1 \cdot \operatorname{sp} \rho_{5}(B)=\left\{[-1]^{1}\right\}$,

giving $\operatorname{sp}\left(P_{4}\right)$ as claimed in (6).

Now to find the spectra of the different quotient graphs, induced from each partition, from Theorem 1.2 and Lemma 3.1. we need to know the ranks of $\rho_{i}\left(H_{j}\right)$ for all group stabilizers $H_{j}$. With this aim, we use the matrices of all irreducible representation shown in Table 3. This gives:

(i) $(4=4)$ :

$H_{1}=\operatorname{Stab}_{S_{4}}(\{1,2,3,4\})=S_{4}$ and so $\rho_{i}\left(H_{1}\right)=\sum_{g \in S_{4}} \rho_{i}(g)$ for $i=1, \ldots, 5$.

(ii) $(4=3+1)$ :

$H_{2}=\operatorname{Stab}_{S_{4}}(\{1,2,3\}) \cap \operatorname{Stab}_{S_{4}}(4)=S_{3}$ and so $\rho_{i}\left(H_{2}\right)=\sum_{g \in S_{3}} \rho_{i}(g)$ for $i=1, \ldots, 5$.

(iii) $(4=2+2)$ :

$H_{3}=\operatorname{Stab}_{S_{4}}(\{1,2\}) \cap \operatorname{Stab}_{S_{4}}(\{3,4\})=\{e,(12),(34),(12)(34)\}$ and so $\rho_{i}\left(H_{3}\right)=$ $\sum_{g \in H_{3}} \rho_{i}(g)$ for $i=1, \ldots, 5$. 


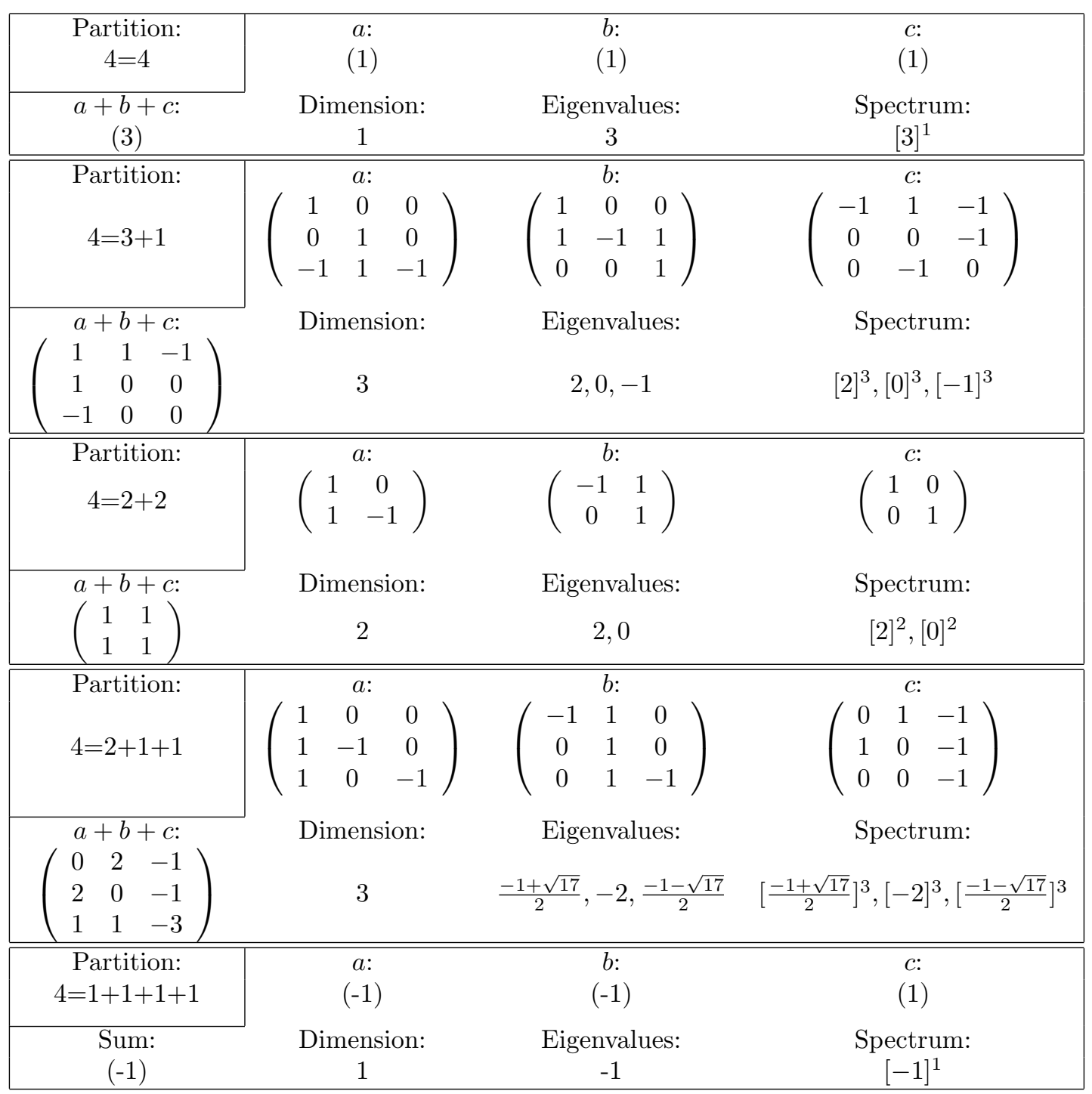

Table 2: The irreducible matrices of $P(4)$, their sum, and their corresponding eigenvalues.

(iv) $(4=2+1+1)$ :

$H_{4}=\operatorname{Stab}_{S_{4}}(\{1,2\}) \cap \operatorname{Stab}_{S_{4}}(3) \cap \operatorname{Stab}_{S_{4}}(4)=\{e,(12)\}$ and so $\rho_{i}\left(H_{4}\right)=\rho_{i}(e)+$ $\rho_{i}((12))$ for $i=1, \ldots, 5$.

(v) $(4=1+1+1+1)$ :

$H_{5}=\operatorname{Stab}_{S_{4}}(1) \cap \operatorname{Stab}_{S_{4}}(2) \cap \operatorname{Stab}_{S_{4}}(3) \cap \operatorname{Stab}_{S_{4}}(4)=\{e\}$ and so $\rho_{i}\left(H_{5}\right)=\rho_{i}(e)=d_{\rho_{i}}$ for $i=1, \ldots, 5$.

The ranks for the cases $(i)-(v)$, together with the corresponding spectra, are shown in Table 
4. Notice that, in the last but one row of the same table, we have the spectrum of the whole graph $P(4)$ again.

Example (New mixed graph $\Gamma(3,3,2))$. Consider the mixed graph $\Gamma(3,3,2)$ again. Now, by using Theorem 1.1, the spectrum of the whole graph is the union of the following spectra:

(i) $1 \cdot \operatorname{sp} \rho_{1}(B)=\left\{[3]^{1}\right\}$,

(ii) $3 \cdot \operatorname{sp} \rho_{2}(B)=\left\{[1]^{1},[-1]^{1}\right\}$,

(iii) $2 \cdot \operatorname{sp} \rho_{3}(B)=\left\{[ \pm \sqrt{3}]^{1}\right\}$,

(iv) $3 \cdot \operatorname{sp} \rho_{4}(B)=\left\{[1]^{2},[-1]^{1}\right\}$,

(v) $1 \cdot \operatorname{sp} \rho_{5}(B)=\left\{[-3]^{1}\right\}$,

which gives (7). Then, the spectra of the different quotient graphs, induced from each partition, are again computed by using the ranks of $\rho_{i}\left(H_{j}\right)$ for the group stabilizers $H_{j}$, as in the previous example for $P(4)$. The obtained results are shown in Table 5, where we indicate the spectrum of the whole new mixed graph in the last but one row.

\section{References}

[1] S. B. Akers and B. Krishnamurthy, A group-theoretic model for symmetric interconnection networks, IEEE Trans. Comput. 38 (1989), no. 4, 555-566.

[2] M. Burrow, Representation Theory of Finite Groups, Dover, New York, 1993.

[3] J. Cibulka, On average and highest number of flips in pancake sorting, Theor. Comput. Sci. 412 (2011) 822-834.

[4] F. Comellas and M. A. Fiol, Vertex-symmetric digraphs with small diameter, Discrete Appl. Math. 58 (1995), no. 1, 1-12.

[5] C. Dalfó, A new general family of mixed graphs, Discrete Appl. Math (2019), in press, doi.org/10.1016/j.dam.2018.12.016.

[6] C. Dalfó, M. A. Fiol, M. Miller, J. Ryan, and J. Širáň, An algebraic approach to lifts of digraphs, Discrete Appl. Math. (2019), in press,

doi.org/10.1016/j.dam.2018.10.040.

[7] C. Dalfó, M. A. Fiol, and J. Širáň, The spectra of lifted digraphs, J. Algebraic Combin. (2019), in press, doi.org/10.1007/s10801-018-0862-y.

[8] C. Dalfó, M. A. Fiol, S. Pavlíková, and J. Širáň, Spectra and eigenspaces of arbitrary lifts of graphs, submitted (2019). 
[9] H. Dweighter, Elementary problems and solutions, problem E2569, Amer. Math. Monthly 82 (1975), no. 10, 1010.

[10] V. Faber, J. W. Moore, and W. Y. C. Chen, Cycle prefix digraphs for symmetric interconnection networks, Networks 23 (1993) 641-649.

[11] W. H. Gates and C. H. Papadimitriou, Bounds for sorting by prefix reversal, Discrete Math. 27 (1979) 47-57.

[12] C. D. Godsil, Algebraic Combinatorics, Chapman and Hall, New York, 1993.

[13] J. L. Gross and T. W. Tucker, Generating all graph coverings by permutation voltage assignments, Discrete Math. 18 (1977) 273-283.

[14] M. H. Heydari and I. H. Sudborough, On the diameter of the pancake network, $J$. Algorithms 25 (1997) 67-94.

[15] G. James and M. Liebeck, Representations and Characters of Groups, 2nd ed., Cambridge Univ. Press, 2001.

[16] E. Konstantinova, On some structural properties of star and pancake graphs, in Information Theory, Combinatorics, and Search Theory, 472-487, Lecture Notes in Comput. Sci. 7777, Springer, Heidelberg, 2013.

[17] M. Miller and J. Širáň, Moore graphs and beyond: A survey of the degree/diameter problem, Electron. J. Combin. 20(2) (2013) \#DS14v2.

[18] N. J. A. Sloane, The on-line encyclopedia of integer sequences, A058986, https://oeis.org. 


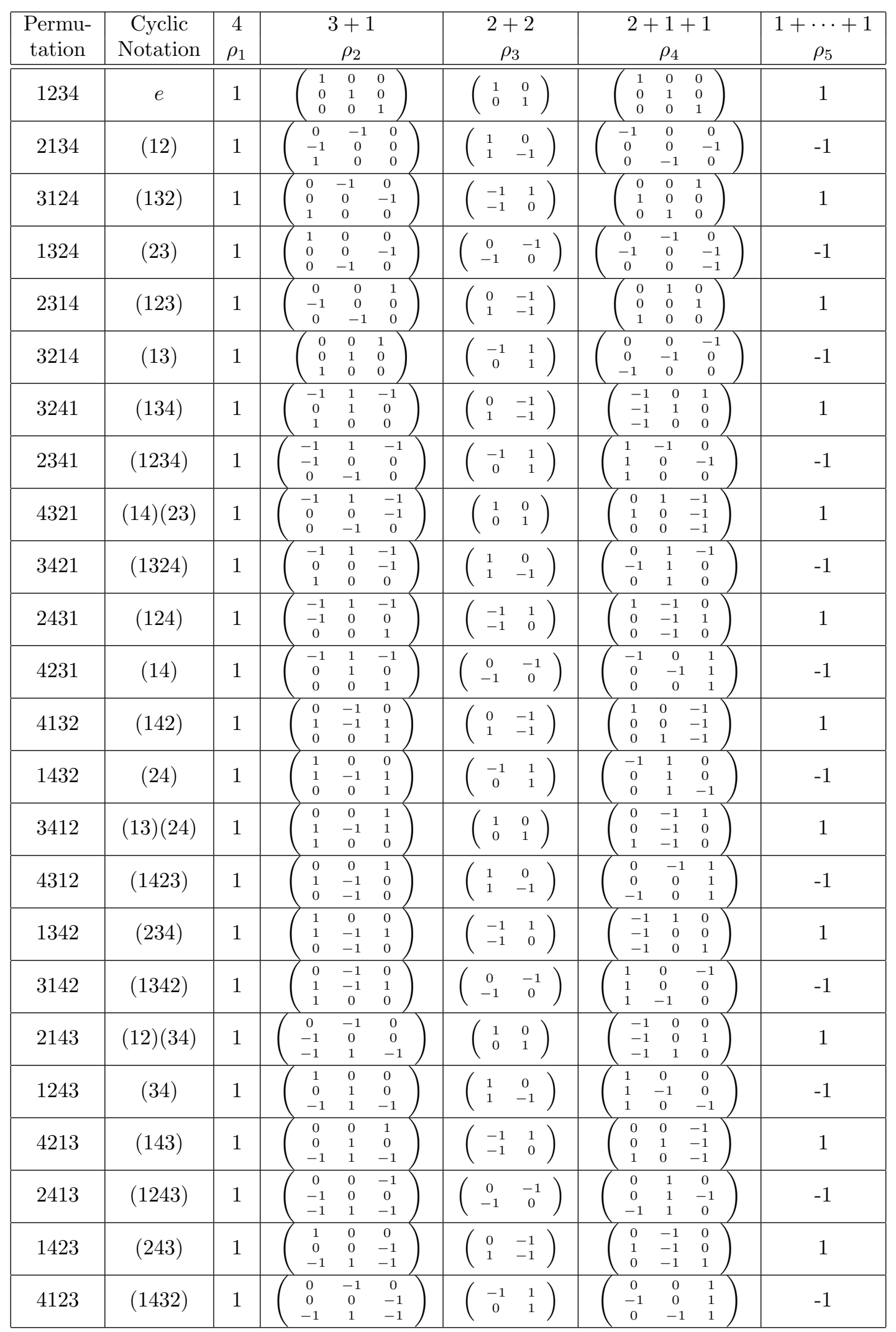

Table 3: The irreducible representations of the symmetric group $S_{4}$. 


\begin{tabular}{|c|c|c|c|c|c|c|}
\hline & $\begin{array}{r}4 \\
\rho_{1} \\
\end{array}$ & $\begin{array}{c}+1 \\
\rho_{2} \\
\end{array}$ & $\begin{array}{c}2+2 \\
\rho_{3} \\
\end{array}$ & $\begin{array}{c}2+1+1 \\
\rho_{4} \\
\end{array}$ & $\begin{array}{c}1+1+1+1 \\
\rho_{5} \\
\end{array}$ & Spectrum \\
\hline 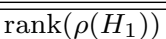 & $\overline{1}$ & 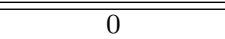 & $\overline{\overline{0}}$ & $\overline{00}$ & $\overline{00}$ & $\left\{[3]^{1}\right\}$ \\
\hline $\operatorname{rank}\left(\rho\left(H_{2}\right)\right)$ & 1 & 1 & 0 & 0 & 0 & $\begin{array}{c}\left\{[3]^{1},[2]^{1},[0]^{1}\right. \\
\left.[-1]^{1}\right\}\end{array}$ \\
\hline $\operatorname{rank}\left(\rho\left(H_{3}\right)\right)$ & 1 & 1 & 1 & 0 & 0 & $\begin{array}{c}\left\{[3]^{1},[2]^{2},[0]^{2},\right. \\
\left.[-1]^{1}\right\}\end{array}$ \\
\hline $\operatorname{rank}\left(\rho\left(H_{4}\right)\right)$ & 1 & 2 & 1 & 1 & 0 & $\begin{array}{c}\left\{[3]^{1},[2]^{3},[0]^{3}\right. \\
{\left[\frac{-1 \pm \sqrt{17}}{2}\right]^{1}} \\
\left.[-1]^{2},[-2]^{1}\right\}\end{array}$ \\
\hline $\operatorname{rank}\left(\rho\left(H_{5}\right)\right)$ & 1 & 3 & 2 & 3 & 1 & $\begin{array}{c}\left\{[3]^{1},[2]^{5},[0]^{5}\right. \\
{\left[\frac{-1 \pm \sqrt{17}}{2}\right]^{3}} \\
\left.[0]^{5},[-1]^{4},[-2]^{3}\right\}\end{array}$ \\
\hline$\overline{\operatorname{sp} \rho(B)}$ & 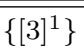 & $\begin{array}{c}\left\{[2]^{1},[0]^{1},[-1]^{1}\right\} \\
\end{array}$ & $\left\langle[2]^{1},[0]^{1}\right\}$ & $\bar{c}\left\{\left[\frac{-1 \pm \sqrt{17}}{2}\right]^{1},[-2]^{1}\right\}$ & 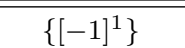 & \\
\hline
\end{tabular}

Table 4: Spectra of the quotient graphs of $P(4)$.

\begin{tabular}{|c|c|c|c|c|c||c|}
\hline & 4 & $3+1$ & $2+2$ & $2+1+1$ & $1+1+1+1$ & Spectrum \\
& $\rho_{1}$ & $\rho_{2}$ & $\rho_{3}$ & $\rho_{4}$ & $\rho_{5}$ & $\left\{[3]^{1}\right\}$ \\
\hline \hline $\operatorname{rank}\left(\rho\left(H_{1}\right)\right)$ & 1 & 0 & 0 & 0 & 0 & $\left\{[3]^{1},[1]^{2},[-1]^{1}\right\}$ \\
\hline $\operatorname{rank}\left(\rho\left(H_{2}\right)\right)$ & 1 & 1 & 0 & 0 & 0 & $\left\{[3]^{1},[ \pm \sqrt{3}]^{1},[1]^{2},[-1]^{1}\right\}$ \\
\hline $\operatorname{rank}\left(\rho\left(H_{3}\right)\right)$ & 1 & 1 & 1 & 0 & 0 & $\left\{[3]^{1},[ \pm \sqrt{3}]^{1},[1]^{5},[-1]^{4}\right\}$ \\
\hline $\operatorname{rank}\left(\rho\left(H_{4}\right)\right)$ & 1 & 2 & 1 & 1 & 0 & $\left\{[3]^{1},[ \pm \sqrt{3}]^{2},[1]^{9},[-1]^{9},[-3]^{1}\right\}$ \\
\hline $\operatorname{rank}\left(\rho\left(H_{5}\right)\right)$ & 1 & 3 & 2 & 3 & 1 & \\
\hline \hline $\operatorname{sp} \rho(B)$ & $\left\{[3]^{1}\right\}$ & $\left\{[1]^{1},[-1]^{2}\right\}$ & $\left\{[ \pm \sqrt{3}]^{1}\right\}$ & $\left\{[1]^{2},[-1]^{1}\right\}$ & $\left\{[-3]^{1}\right\}$ & \\
\hline
\end{tabular}

Table 5: Spectra of the quotient graphs of $\Gamma(3,3,2)$. 